\title{
Myasthenic Crisis
}

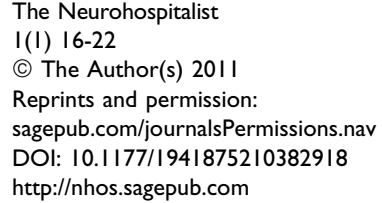

(S)AGE

\section{Linda C. Wendell, MD', and Joshua M. Levine, $M D^{2}$}

\begin{abstract}
Myasthenic crisis is a complication of myasthenia gravis characterized by worsening of muscle weakness, resulting in respiratory failure that requires intubation and mechanical ventilation. Advances in critical care have improved the mortality rate associated with myasthenic crisis. This article reviews the epidemiology of myasthenic crisis and discusses patient evaluation. Therapeutic options including mechanical ventilation and pharmacological and surgical treatments are also discussed.
\end{abstract}

\section{Keywords}

myasthenia gravis, autoimmune diseases of the nervous system, neurocritical care clinical specialty, neuromuscular disease clinical specialty

Myasthenia gravis (MG) is an autoimmune disorder affecting neuromuscular transmission, leading to generalized or localized weakness characterized by fatigability. ${ }^{1}$ It is the most common disorder of the neuromuscular junction, with an annual incidence of $0.25-2$ patients per $100000 .^{2}$ Myasthenia gravis is most frequently associated with antibodies against acetylcholine receptors (AChR) in the post-synaptic motor end plate. ${ }^{3}$ A second form of myasthenia gravis, usually seen in young women, involves antibodies against muscle-specific tyrosine kinase (MuSK). ${ }^{4}$ A third group of patients has antibodies to neither AChR nor MuSK, and these patients are considered seronegative. Clinically, these patients are similar to patients with AChR antibodies. ${ }^{3}$ Myasthenic crisis is a complication of MG characterized by worsening muscle weakness, resulting in respiratory failure that requires intubation and mechanical ventilation. ${ }^{1}$ A more comprehensive definition of myasthenic crisis also includes post-surgical patients, in whom exacerbation of muscle weakness from MG causes a delay in extubation. ${ }^{5}$

\section{Epidemiology}

Fifteen to $20 \%$ of myasthenic patients are affected by myasthenic crisis at least once in their lives. ${ }^{1}$ The median time to first myasthenic crisis from onset of MG ranges from 8-12 months. ${ }^{6,7}$ However, myasthenic crisis may be the initial presentation of MG in one-fifth of patients. ${ }^{7,8}$ Overall, women are twice as likely as men to be affected. A bimodal distribution of myasthenic crisis is seen. An early peak prior to age 55 affects women $4: 1$, whereas a later peak after age 55 affects women and men equally. ${ }^{6}$ The average age of admission with myasthenic crisis is almost 59 years. Patients in crisis requiring endotracheal intubation spend a median of 17 days in the hospital. Eighteen percent of patients admitted with myasthenic crisis will require discharge to a rehabilitation center. ${ }^{9}$

Advances in mechanical ventilation and critical care have been paramount in improving mortality associated with myasthenic crisis. During the early 1960s, respiratory care of these patients was transitioned from negative external pressure ventilation to positive pressure ventilation in an intensive care unit. The mortality rate from myasthenic crisis declined from $42 \%$ in the early 1960 s to $6 \%$ by the late $1970 \mathrm{~s}$, and the median age at death increased. ${ }^{10}$ Currently, mortality is $4 \%$ and is primarily the result of comorbid medical conditions. ${ }^{6}$

\section{Assessment of Respiratory Dysfunction in Myasthenic Crisis}

Myasthenic crisis can involve the upper airway muscles, respiratory muscles, or a combination of both muscle groups. ${ }^{11}$ Both inspiratory and expiratory respiratory muscles can be affected, manifesting as dyspnea. ${ }^{12}$ Inspiration is performed primarily by the diaphragm and external intercostal muscles and secondarily by the sternocleidomastoid and scalene muscles. Although expiration is primarily passive, the abdominal and internal intercostal muscles can be recruited to assist. ${ }^{11}$ In MG with AChR antibodies, muscle weakness

\footnotetext{
'Department of Neurology, University of Pennsylvania, Philadephia, PA

${ }^{2}$ Departments of Neurology, Neurosurgery, and Anesthesiology and Critical Care, University of Pennsylvania, Philadelphia, PA

Corresponding Author:

Joshua M. Levine, MD, Hospital of the University of Pennsylvania, Department of Neurology, 3 West Gates, 3400 Spruce Street, Philadelphia, PA 19104

Email: Joshua.Levine@uphs.upenn.edu
} 
tends to initially affect the intercostal and accessory muscles and then the diaphragm. ${ }^{3}$

Inspiratory function is measured by both vital capacity (VC) and negative inspiratory force (NIF); expiratory function is measured by positive expiratory force (PEF). ${ }^{11} \mathrm{~A}$ VC less than $1 \mathrm{~L}$ (or $<20-25 \mathrm{~mL} / \mathrm{kg}$ ) or an NIF $<20 \mathrm{~cm} \mathrm{H} 2 \mathrm{O}$ indicates significant respiratory weakness; both measurements are commonly used to define myasthenic crisis. ${ }^{13,14}$ In addition, a PEF $<40 \mathrm{~cm} \mathrm{H} 2 \mathrm{O}$ may indicate crisis. ${ }^{15}$ Regardless of respiratory function indices, the need for mechanical ventilation is a sufficient criterion to define myasthenic crisis. ${ }^{13}$

Inspiratory and expiratory forces are more sensitive than $\mathrm{VC}$ in evaluating muscle strength in $\mathrm{MG}^{12}$ In a retrospective review, repeated measurement of $\mathrm{VC}$ did not predict the need for intubation and mechanical ventilation in myasthenic crisis. ${ }^{16}$ Facial weakness can lead to inaccurate measurements of all 3 indices. ${ }^{15}$ At the bedside, recruitment of accessory muscles indicates significant inspiratory muscle weakness, and a weak cough or difficulty counting to 20 in a single breath signifies weakness of the expiratory muscles. ${ }^{11}$

Respiratory dysfunction also manifests as upper airway obstruction if weakness of the upper airway or bulbar muscles is present. ${ }^{17}$ Myasthenic patients with MuSK antibodies preferentially exhibit bulbar weakness before respiratory muscle weakness. ${ }^{3}$ Upper airway weakness can lead to respiratory failure by oropharyngeal collapse or tongue obstruction and by increasing the work of already fatigued respiratory muscles against a closed airway. ${ }^{17}$ Signs of bulbar weakness include dysphagia, nasal regurgitation, a nasal quality to speech, staccato speech, jaw weakness (jaw closure weaker than jaw opening), bifacial paresis, and tongue weakness. ${ }^{14}$

\section{Respiratory Management of Myasthenic Crisis}

\section{Intubation and Mechanical Ventilation}

Respiratory support is imperative in the management of myasthenic crisis. Two-thirds to $90 \%$ of patients with myasthenic crisis require intubation and mechanical ventilation. ${ }^{6,8}$ Over $20 \%$ of patients require intubation during evaluation in the emergency department, and almost $60 \%$ are intubated after admission to an intensive care unit. ${ }^{6}$ Elective intubation of a myasthenic patient with impending respiratory failure is favored over emergent intubation. Once intubated, patients should be placed on an assisted ventilator setting with tidal volumes of $8-10 \mathrm{cc} / \mathrm{kg}$ ideal body weight and pressure support of $8-15 \mathrm{~cm} \mathrm{H}_{2} \mathrm{O}$ to prevent atelectasis and to minimize the work of breathing. The degree of support required is ultimately patient dependent. ${ }^{18}$

Neuromuscular blocking agents (paralytics) should be used with caution when intubating MG patients. Depolarizing agents (for example, succinylcholine) are less potent in myasthenics because fewer functional post-synaptic AChR are available. This decrease in receptors also results in a decrease in the safety margin or remaining AChR available for neuromuscular transmission. Nondepolarizing agents (for example, vecuronium) have increased potency, and reduced doses are required for paralysis. ${ }^{19}$

Weaning from the ventilator should be initiated after the patient demonstrates clinical improvement, typically at a vital capacity of more than $15 \mathrm{~mL} / \mathrm{kg} .{ }^{18,20}$ Improvement in the strength of neck flexors and other adjunct muscles usually is associated with improvement in bulbar and respiratory muscle strength and can be a useful tool for assessing clinical improvement. ${ }^{20}$ Patients should be transitioned to a spontaneous mode of ventilation (eg, pressure support ventilation) in which all breaths are patient initiated. Pressure support can then gradually be decreased to minimal settings. If the patient does not tolerate weaning, an assisted ventilator setting should be reinstituted. ${ }^{18}$

It remains unclear when first to attempt extubation after myasthenic crisis. Only half of patients are extubated at 13 days. ${ }^{6}$ In 1 series, 3 independent risk factors for prolonged intubation ( $>14$ days) were identified: age $>50$ years, peak vital capacity $<25 \mathrm{~mL} / \mathrm{kg}$ on post-intubation days 1 to 6 , and a serum bicarbonate $\geq 30 \mathrm{mmol} / \mathrm{L}$. All of the patients with no risk factors were intubated for less than 2 weeks, whereas $88 \%$ of the patients with all 3 risk factors had prolonged intubation. Patients with a prolonged intubation were hospitalized 3 times longer and were less likely to be functionally independent upon discharge. ${ }^{6}$ Thymoma is also associated with a worse prognosis in myasthenic crisis. ${ }^{8}$

Fluctuating weakness and pulmonary complications often confound the decision to extubate. ${ }^{21} \mathrm{~A}$ maximal expiratory pressure has been demonstrated to independently predict extubation success. Extubation failure is most commonly associated with a weak cough and inadequate airway clearance. ${ }^{22}$ Older age, atelectasis, and pneumonia are also associated with extubation failure. ${ }^{7}$ Tracheostomy placement ranges from $14 \%-40 \%{ }^{6,7}$

Reintubation occurs more than one-fourth of the time., ${ }^{7,21}$ Acidosis, decreased forced vital capacity (FVC), atelectasis, and need for noninvasive ventilatory support are predictors of reintubation. ${ }^{21}$ Two retrospective studies found atelectasis in all patients requiring reintubation. ${ }^{7,21}$ To prevent atelectasis, early intubation, aggressive chest physiotherapy, and frequent suctioning should be implemented and high positive end-expiratory pressure given while the patient is mechanically ventilated. ${ }^{23}$ Reintubaton is a significant event because patients requiring reintubation have significantly longer ICU and hospital stays. ${ }^{7}$

\section{Noninvasive Ventilation}

Noninvasive ventilation (NIV) may be used to prevent intubation or reintubation of patients in myasthenic crisis. ${ }^{22}$ With bilevel positive airway pressure (BiPAP), positive pressure is applied during both phases of respiration, enhancing airflow and alleviating the work of breathing during inspiration and 
Table I. Precipitants of Myasthenic Crisis

\begin{tabular}{ll}
\hline Stressors & Medications \\
\hline Physical stressors & $\alpha$-Interferon \\
Aspiration pneumonitis & Antibiotics \\
Infection & Aminoglycosides \\
Perimenstrual state & Gentamicin \\
Pregnancy & Streptomycin \\
Sleep deprivation & Ampicillin \\
Surgery & Macrolides \\
Environmental Stressors & Erythromycin \\
Emotional stress & Quinolones \\
Pain & Ciprofloxacin \\
Temperature extremes & Polymyxin \\
Tapering of immune-modulating & Antiepileptics \\
medications & Gabapentin \\
& Phenytoin \\
& $\beta$-Adrenergic antagonists \\
& Calcium channel antagonists \\
& Contrast media \\
& Magnesium \\
& Methimazole \\
\hline & Prednisone \\
& Procainamide \\
\hline
\end{tabular}

preventing airway collapse and atelectasis during expiration. ${ }^{24}$ One retrospective study found that $20 \%$ of patients in myasthenic crisis could be successfully supported with NIV. In patients who are initially managed with NIV, endotracheal intubation and mechanical ventilation should be initiated if the patient has continued or worsening shortness of breath, increased work of breathing, tachypnea, or hypercapnea. Independent predictors of NIV success are a serum bicarbonate $<30 \mathrm{mmol} / \mathrm{L}$ and an APACHE II score $<6 .{ }^{22} \mathrm{An}$ independent predictor of NIV failure is hypercapnia $(\mathrm{PCO} 2>45 \mathrm{~mm} \mathrm{Hg}){ }^{24}$ Vital capacity, NIF, and PEF are not useful in predicting outcome. ${ }^{25}$

Initial use of NIV is associated with a shorter duration of ventilatory support. Patients treated initially with NIV require ventilatory support for a median of 4 days versus 9 days in those patients initially intubated. In addition, these patients spend one-third less time in the ICU and in the hospital. ${ }^{24}$ In 2 studies, NIV prevented reintubation in $70 \%$ of patients. ${ }^{22,25}$ In patients with bulbar weakness, NIV might increase the risk of aspiration. ${ }^{22}$ However, 1 retrospective cohort study found no difference in pulmonary complications between those supported with NIV and those supported with endotracheal intubation mechanical ventilation. In patients successfully supported with $\mathrm{NIV}$, there were significantly fewer pulmonary complications. ${ }^{24}$

\section{Complications in the Management of Myasthenic Crisis}

Fever is the most common complication associated with myasthenic crisis. Infectious complications include pneumonia, bronchitis, urinary tract infections, Clostridium difficile
Table 2. Symptoms of Cholinergic Crisis

colitis, bacteremia, and sepsis. ${ }^{6}$ When compared to patients admitted for non-crisis MG, patients admitted with myasthenic crisis are more likely to experience sepsis, deep vein thrombosis, and cardiac complications including congestive heart failure, acute myocardial infarction, arrhythmias, and cardiac arrest. These complications, however, are not independent predictors of mortality. ${ }^{9}$ In 1 series, atelectasis, C. difficile colitis, transfusion-dependent anemia, and congestive heart failure were independently associated with a longer duration of myasthenic crisis, but not with a longer duration of intubation. ${ }^{6}$

\section{Precipitants of Myasthenic Crisis}

Common precipitants of myasthenic crisis are shown in Table 1. The most common precipitant is infection. ${ }^{6,8}$ One series documented infection in $38 \%$ of patients presenting with myasthenic crisis; most commonly, the infection was bacterial pneumonia followed by a bacterial or viral upper respiratory infection. ${ }^{6}$ Other precipitants include aspiration pneumonitis, surgery, pregnancy, perimenstrual state, certain medications (see below), and tapering of immune-modulating medications. ${ }^{6,8,11}$ Other antecedent factors include exposure to temperature extremes, pain, sleep deprivation, and physical or emotional stress. ${ }^{26}$ Approximately one-third to one-half of patients may have no obvious cause for their myasthenic crisis. ${ }^{6,8}$

Numerous medications may exacerbate MG, including quinidine,${ }^{27}$ procainamide, ${ }^{28} \beta$-adrenergic antagonists ${ }^{29}$ calcium channel antagonists (verapamil, nifedipine, felodipine), ${ }^{30-32}$ magnesium, ${ }^{33}$ antibiotics (ampicillin, gentamicin, streptomycin, polymyxin, ciprofloxacin, erythromycin), ${ }^{34-38}$ phenytoin, ${ }^{39}$ gabapentin, ${ }^{40}$ methimazole, ${ }^{41} \alpha$-interferon, ${ }^{42}$ and contrast media. ${ }^{43}$ These medications should be used cautiously in myasthenic patients, especially after surgery. Any medication suspected of precipitating myasthenic crisis should be discontinued. ${ }^{26}$

Although corticosteroids can be used in the treatment of MG, initial treatment with prednisone led to an exacerbation of MG in almost half of patients in 1 series. ${ }^{44}$ The incidence of myasthenic crisis resulting from corticosteroids ranges from $9 \%-18 \% .^{44,45}$ Thus, commencement of corticosteroids for the treatment of MG should always occur in a hospital 
Table 3. Comparison of Intravenous Immunoglobulin to Plasma Exchange

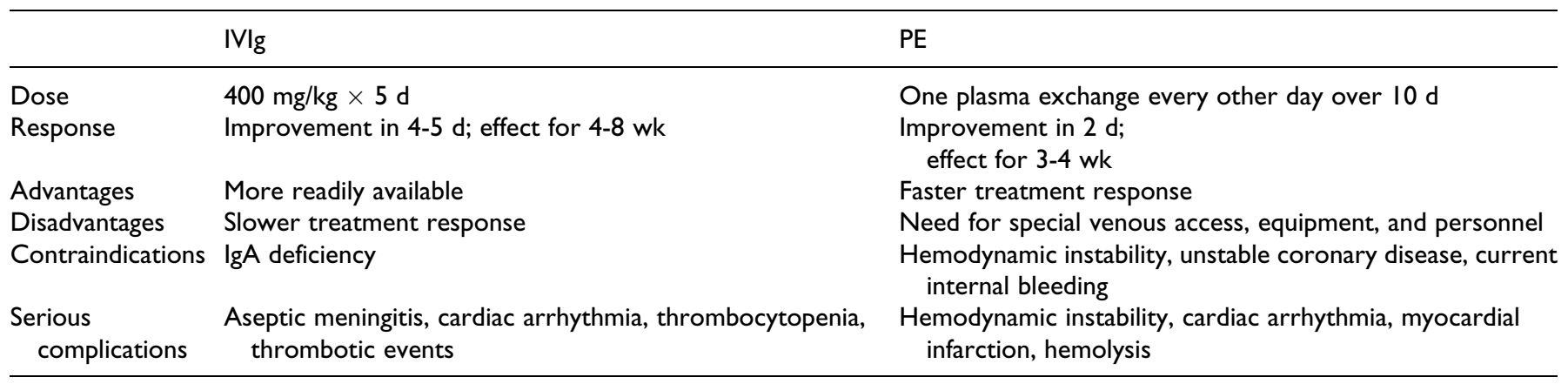

Abbreviations: IVlg, intravenous immunoglobulin; PE, plasma exchange.

setting, where respiratory function can be monitored. ${ }^{44}$ Predictors of exacerbation from prednisone include older age, lower score on the Myasthenia Severity Scale (a clinical assessment tool), and bulbar symptoms. ${ }^{45}$

\section{Cholinergic Crisis}

Patients taking an excess of acetylcholinesterase inhibitors may precipitate a cholinergic crisis characterized by both muscarinic and nicotinic toxicity (Table 2). ${ }^{15}$ Symptoms may include an increase in perspiration, lacrimation, salivation and pulmonary secretions, nausea, vomiting, diarrhea, bradycardia, and fasciculations. ${ }^{11,15}$ Although cholinergic crisis is an important consideration in the evaluation of the patient in myasthenic crisis, it is uncommon. ${ }^{15}$ One retrospective series of patients with myasthenic crisis found none of the patients had cholinergic crisis. ${ }^{6}$ Regardless of whether myasthenic or cholinergic crisis is suspected, acetylcholinesterase inhibitors should be significantly lowered or discontinued to avoid excessive pulmonary secretions in the setting of respiratory distress. ${ }^{11}$

\section{Treatment of Myasthenic Crisis}

The 2 primary pharmacologic therapies available for myasthenic crisis are intravenous immunoglobulin (IVIg) and plasma exchange (PE) (Table 3). A typical course of IVIg is $400 \mathrm{mg} / \mathrm{kg}$ daily for 5 days. ${ }^{13}$ Patients should be screened for IgA deficiency to avoid anaphylaxis from IVIg. ${ }^{46}$ The most common side effect associated with IVIg is headache. Other complications include fever, nausea, IV site discomfort, rash, malaise, aches and pain. More serious adverse events can include aseptic meningitis, hypertension, cardiac arrhythmia, thrombocytopenia, and thrombotic events, including stroke, myocardial infarction, and pulmonary embolism. ${ }^{47,48}$ For PE, 5 exchanges (1 plasma volume or 3-4 L per exchange) are usually performed every other day over 10 days. ${ }^{13,49}$ Replacement fluid is generally a solution of normal saline $/ 5 \%$ albumin. Venous access is obtained via large-bore peripheral catheters, or temporary or tunneled double-lumen central venous catheters. Infection and bleeding from obtaining central venous access occurs in less than $2 \%{ }^{49}$ Common complications from PE include fever, symptoms from hypocalcemia (primarily paresthesias), a transient decrease in blood pressure, and tachycardia. ${ }^{49,50}$ Other more serious, but less common, adverse effects include cardiac arrhythmia, myocardial infarction, and hemolysis. ${ }^{50}$ Response to treatment generally occurs after 2 days with PE and after 4-5 days with IVIg. ${ }^{51}$ For both treatments, an effect can be seen for several weeks. ${ }^{20}$ If there is insufficient or no response to treatment, PE can be given after IVIg, and IVIg can be administered after PE. ${ }^{46,52}$ Although there is a theoretical concern that sequential use of PE after IVIg might result in removal of the IVIg, removal is usually done when there has been insufficient response to IVIg, and removal of IVIg is therefore of little concern.

Some evidence exists that PE may be more effective than IVIg in the treatment of myasthenic crisis. A retrospective multicenter study including only patients experiencing myasathenic crisis compared the use of 5 or 6 cycles of PE completed every other day to $400 \mathrm{mg} / \mathrm{kg} /$ day of IVIg given for 5 days and found PE to be more effective. Patients who initially received PE had more clinical improvement at 1 week, better respiratory status at 2 weeks, and better functional outcome at 1 month. However, an increased number of complications, mostly infection and cardiovascular instability, were seen in the PE group. ${ }^{53}$ Conversely, another study prospectively randomized patients with an exacerbation of MG to 3 cycles of PE or to IVIg $400 \mathrm{mg} / \mathrm{kg} /$ day given for 3 or 5 days and found no difference. However, this study was not limited to patients in myasthenic crisis. ${ }^{54}$ Overall, one-fifth of patients required a second treatment with either PE or IVIg. Patients who received IVIg as initial treatment more frequently required a second treatment, primarily owing to an absence of early response. ${ }^{53}$ In a case series of 4 patients in myasthenic crisis who had failed IVIg, all improved after PE was implemented. ${ }^{52}$

Corticosteroids are used in conjunction with IVIg and PE. High-dose prednisone $(60-100 \mathrm{mg}$ daily or $1-1.5 \mathrm{mg} / \mathrm{kg} / \mathrm{d}$ ) may be initiated concurrently with IVIg or PE, since prednisone begins to work after 2 weeks, at which point the effects of PE and IVIg are waning. Enteral administration of corticosteroids is preferred, and initiation of prednisione may be deferred until after the patient is extubated if the patient is 
rapidly improving with IVIg or PE treatment. ${ }^{15}$ Initial worsening from high-dose prednisone is treated with continued ventilatory support. ${ }^{26}$ The mean time to improvement with prednisone in 1 series of patients with MG exacerbation was 13 days. Eighty-five percent of patients showed improvement within 3 weeks. Worsening of symptoms with the initiation of corticosteroids is not predictive of overall response to corticosteroids. ${ }^{44}$ Once the patient has begun to show improvement, the prednisone dose can be decreased and gradually converted to alternate-day dosing. ${ }^{26}$ The most common side effects from prednisone are a Cushingoid appearance, cataracts, and weight gain. ${ }^{44}$ Infection, poorly controlled diabetes, and severe osteoporosis are relative contraindications to instituting corticosteroids. ${ }^{15}$

Cyclosporine may be considered after initiation of IVIg or PE in patients who cannot tolerate or who are refractory to corticosteroids. However, the onset of action of cyclosporine is 1-2 months. Other immunomodulating agents, azathioprine and mycophenolate, are not useful in myasthenic crisis because of their long latency of action. ${ }^{15}$

Abnormal laboratory values that could affect muscle strength should also be corrected. Potassium, magnesium, and phosphate depletion can all exacerbate myasthenic crisis and should be repleted. Hematocrit less than $30 \%$ might affect weakness by decreasing oxygen-carrying capacity. ${ }^{13,18}$ Adequate nutrition is important to avoid a negative energy balance and worsening of muscle strength. ${ }^{18}$

As mentioned previously, acetylcholinesterase inhibitors are usually discontinued in myasthenic crisis to avoid excessive bronchial secretions. ${ }^{11}$ Additionally, continued use of these medications confounds the assessment of other treatment modalities and can increase muscle weakness if used in excess. ${ }^{13}$ These medications do not alter the course of the crisis and offer solely symptomatic relief of MG. ${ }^{11}$ After patients have shown definitive improvement in muscle strength (usually several days after the initiation of IVIg or PE), acetylcholinesterase inhibitors, typically oral pyridostigmine, may be reinitiated after or just prior to extubation. ${ }^{11,13}$ Oral pyridostigmine is preferred, but it may be given intravenously. One milligram of intravenous pyridsotigmine is equal to $30 \mathrm{mg}$ of oral pyridostigmine. Patients should be started on a low dose of the medication that is titrated to symptomatic relief. ${ }^{13}$ Continuous intravenous infusion of pyridostigmine as treatment for myasthenic crisis may have efficacy similar to that of plasma exchange, but it is associated with an increased risk of life-threatening cardiac arrhythmia that warrants further study. ${ }^{55,56}$

\section{Thymectomy}

Thymic hyperplasia is common in young myasthenic patients with positive AChR antibodies, especially women. Thymic tumors, found in $15 \%$ of patients with $\mathrm{MG}$ and in $32 \%$ of patients with myasthenic crisis, should be treated with thymectomy. ${ }^{3,6}$ Patients with non-thymomatous MG can consider thymectomy to improve the likelihood of improvement or remission of the disease.$^{57}$ One retrospective study found that myasthenic patients who had undergone thymectomy had fewer incidences of myasthenic crisis and less-severe episodes. ${ }^{58}$ A multicenter, single-blind, randomized controlled trial is currently investigating the benefit of thymectomy in non-thymomatous $\mathrm{MG}^{59}$

Postoperative myasthenic crisis is common after thymectomy; the incidence ranges from $12 \%$ to $34 \% .^{60,61}$ Postoperative crisis in these patients has been related to a history of myasthenic crisis, preoperative presence of bulbar weakness, preoperative serum AChR antibody levels $>100 \mathrm{nmol} / \mathrm{L}$, and intraoperative blood loss of $>1 \mathrm{~L}^{.61}$

\section{Conclusions}

Myasthenic crisis is a common complication of MG. The advent of positive pressure ventilation in the 1960s has decreased mortality and remains the cornerstone of management. The majority of patients with myasthenic crisis require endotracheal intubation and mechanical ventilation. A select group of patients might benefit from NIV to avoid initial intubation or reintubation.

Factors precipitating myasthenic crisis should be quickly identified and promptly mitigated; half of these patients have no identifiable precipitant. Typically, anticholinesterase inhibitors are discontinued to avoid excessive secretions while the patient is experiencing respiratory failure. Both PE and IVIg, in conjunction with prednisone, may be used to treat myasthenic crisis. Limited data suggest that PE may be more effective than IVIg. Thymectomy remains part of treatment in patients with thymic tumors, but the role of surgery in nonthymomatous MG requires further investigation.

\section{Declaration of Conflicting Interests}

The author(s) declared no potential conflicts of interests with respect to the authorship and/or publication of this article.

\section{Financial Disclosure/Funding}

The author(s) received no financial support for the research and/or authorship of this article.

\section{References}

1. Ropper AH, Gress DR, Diringer MN, Green DM, Mayer SA, Bleck TP. Treatment of the Critically Ill Patient With Myasthenia Gravis. Neurological and Neurosurgical Intensive Care. 4th ed. Philadelphia, PA: Lipincott Williams \& Wilkins; 2004:299-311.

2. Vincent A, Palace J, Hilton-Jones D. Myasthenia gravis. Lancet. 2001;357:2122-2128.

3. Chaudhuri A, Behan PO. Myasthenic crisis. QJM. 2009; 102:97-107.

4. Vincent A, Leite MI. Neuromuscular junction autoimmune disease: muscle specific kinase antibodies and treatments for myasthenia gravis. Curr Opin Neurol. 2005;18:519-525. 
5. Bedlack RS, Sanders DB. On the concept of myasthenic crisis. J Clin Neuromuscul Dis. 2002;4:40-42.

6. Thomas CE, Mayer SA, Gungor Y, et al. Myasthenic crisis: clinical features, mortality, complications, and risk factors for prolonged intubation. Neurology. 1997;48:1253-1260.

7. Rabinstein AA, Mueller-Kronast N. Risk of extubation failure in patients with myasthenic crisis. Neurocrit Care. 2005; 3:213-215

8. O'Riordan JI, Miller DH, Mottershead JP, Hirsch NP, Howard RS. The management and outcome of patients with myasthenia gravis treated acutely in a neurological intensive care unit. Eur J Neurol. 1998;5:137-142.

9. Alshekhlee A, Miles JD, Katirji B, Preston DC, Kaminski HJ. Incidence and mortality rates of myasthenia gravis and myasthenic crisis in US hospitals. Neurology. 2009;72:1548-1554.

10. Cohen MS, Younger D. Aspects of the natural history of myasthenia gravis: crisis and death. Ann N Y Acad Sci. 1981; 377:670-677.

11. Juel VC. Myasthenia gravis: management of myasthenic crisis and perioperative care. Semin Neurol. 2004;24:75-81.

12. Mier-Jedrzejowicz AK, Brophy C, Green M. Respiratory muscle function in myasthenia gravis. Am Rev Respir Dis. 1988; 138:867-873.

13. Ahmed S, Kirmani JF, Janjua N, et al. An update on myasthenic crisis. Curr Treat Options Neurol. 2005 Mar;7(2):129-141.

14. Rabinstein AA, Wijdicks EF. Warning signs of imminent respiratory failure in neurological patients. Semin Neurol. 2003;23:97-104.

15. Lacomis D. Myasthenic crisis. Neurocrit Care. 2005;3:189-194.

16. Rieder P, Louis M, Jolliet P, Chevrolet JC. The repeated measurement of vital capacity is a poor predictor of the need for mechanical ventilation in myasthenia gravis. Intensive Care Med. 1995;21:663-668.

17. Putman MT, Wise RA. Myasthenia gravis and upper airway obstruction. Chest. 1996;109:400-404.

18. Kirmani JF, Yahia AM, Qureshi AI. Myasthenic crisis. Curr Treat Options Neurol. 2004;6:3-15.

19. Baraka A. Anaesthesia and myasthenia gravis. Can J Anaesth. 1992;39:476-486.

20. Meriggioli MN. Myasthenia gravis: immunopathogenesis, diagnosis, and management. Continuum: Lifelong Learning in Neurology. 2009;15:35-62.

21. Seneviratne J, Mandrekar J, Wijdicks EF, Rabinstein AA. Predictors of extubation failure in myasthenic crisis. Arch Neurol. 2008;65:929-933.

22. Wu JY, Kuo PH, Fan PC, Wu HD, Shih FY, Yang PC. The role of non-invasive ventilation and factors predicting extubation outcome in myasthenic crisis. Neurocrit Care. 2009;10:35-42.

23. Varelas PN, Chua HC, Natterman J, et al. Ventilatory care in myasthenia gravis crisis: assessing the baseline adverse event rate. Crit Care Med. 2002;30:2663-2668.

24. Seneviratne J, Mandrekar J, Wijdicks EF, Rabinstein AA. Noninvasive ventilation in myasthenic crisis. Arch Neurol. 2008; 65:54-58.
25. Rabinstein A, Wijdicks EF. BiPAP in acute respiratory failure due to myasthenic crisis may prevent intubation. Neurology. 2002;59:1647-1649.

26. Keesey JC. Clinical evaluation and management of myasthenia gravis. Muscle Nerve. 2004;29:484-505.

27. Weisman SJ. Masked myasthenia gravis. J Am Med Assoc. 1949; 141:917.

28. Drachman DA, Skom JH. Procainamide - a hazard in myasthenia gravis. Arch Neurol. 1965;13:316-320.

29. Kaeser HE. Drug-induced myasthenic syndromes. Acta Neurol Scand Suppl. 1984;100:39-47.

30. Rajasekaran D, Chandrasekar S, Rajendran M. Drug related crisis in myasthenia gravis. J Assoc Physicians India. 2006; 54:820-821.

31. Pina Latorre MA, Cobeta JC, Rodilla F, Navarro N, Zabala S. Influence of calcium antagonist drugs in myasthenia gravis in the elderly. J Clin Pharm Ther. 1998;23:399-401.

32. Swash M, Ingram DA. Adverse effect of verapamil in myasthenia gravis. Muscle Nerve. 1992;15:396-398.

33. Bashuk RG, Krendel DA. Myasthenia gravis presenting as weakness after magnesium administration. Muscle Nerve. 1990;13:708-712.

34. Argov Z, Brenner T, Abramsky O. Ampicillin may aggravate clinical and experimental myasthenia gravis. Arch Neurol. 1986;43:255-256.

35. Torda $\mathrm{T}$. The nature of gentamicin-induced neuromuscular block. Br J Anaesth. 1980;52:325-329.

36. Hokkanen E. Antibiotics in myasthenia gravis. Br Med J. 1964; 1:1111-1112.

37. Mumford CJ, Ginsberg L. Ciprofloxacin and myasthenia gravis. Br Med J. 1990;301:818.

38. May EF, Calvert PC. Aggravation of myasthenia gravis by erythromycin. Ann Neurol. 1990;28:577-579.

39. Brumlik J, Jacobs RS. Myasthenia gravis associated with diphenylhydantoin therapy for epilepsy. Can J Neurol Sci. 1974; 1:127-129.

40. Scheschonka A, Beuche W. Treatment of post-herpetic pain in myasthenia gravis: exacerbation of weakness due to gabapentin. Pain. 2003;104:423-424.

41. Kuroda Y, Endo C, Neshige R, Kakigi R. Exacerbation of myasthenia gravis shortly after administration of methimazole for hyperthyroidism. Jpn J Med. 1991;30:578-581.

42. Konishi T. A case of myasthenia gravis which developed myasthenic crisis after alpha-interferon therapy for chronic hepatitis C [in Japanese]. Rinsho Shinkeigaku. 1996;36:980-985.

43. Eliashiv S, Wirguin I, Brenner T, Argov Z. Aggravation of human and experimental myasthenia gravis by contrast media. Neurology. 1990;40:1623-1625.

44. Pascuzzi RM, Coslett HB, Johns TR. Long-term corticosteroid treatment of myasthenia gravis: report of 116 patients. Ann Neurol. 1984;15:291-298.

45. Bae JS, Go SM, Kim BJ. Clinical predictors of steroid-induced exacerbation in myasthenia gravis. J Clin Neurosci. 2006; 13:1006-1010. 
46. Younger DS, Raksadawan N. Therapy in neuromuscular disease. Neurol Clin. 2001;19:205-215, vii.

47. Bertorini TE, Nance AM, Horner LH, Greene W, Gelfand MS, Jaster JH. Complications of intravenous gammaglobulin in neuromuscular and other diseases. Muscle Nerve. 1996; 19:388-391.

48. Grillo JA, Gorson KC, Ropper AH, Lewis J, Weinstein R. Rapid infusion of intravenous immune globulin in patients with neuromuscular disorders. Neurology. 2001;57:1699-1701.

49. Shemin D, Briggs D, Greenan M. Complications of therapeutic plasma exchange: a prospective study of 1,727 procedures. J Clin Apher. 2007;22:270-276.

50. Rodnitzky RL, Goeken JA. Complications of plasma exchange in neurological patients. Arch Neurol. 1982;39:350-354.

51. Gold R, Schneider-Gold C. Current and future standards in treatment of myasthenia gravis. Neurotherapeutics. 2008; 5:535-541.

52. Stricker RB, Kwiatkowska BJ, Habis JA, Kiprov DD. Myasthenic crisis. Response to plasmapheresis following failure of intravenous gamma-globulin. Arch Neurol. 1993; 50:837-840.

53. Qureshi AI, Choudhry MA, Akbar MS, et al. Plasma exchange versus intravenous immunoglobulin treatment in myasthenic crisis. Neurology. 1999;52:629-632.

54. Gajdos P, Chevret S, Clair B, Tranchant C, Chastang C. Clinical trial of plasma exchange and high-dose intravenous immunoglobulin in myasthenia gravis. Myasthenia Gravis Clinical Study Group. Ann Neurol. 1997;41:789-796.

55. Berrouschot J, Baumann I, Kalischewski P, Sterker M, Schneider D. Therapy of myasthenic crisis. Crit Care Med. 1997;25:1228-1235.

56. Mayer SA, Thomas CE. Therapy of myasthenic crisis. Crit Care Med. 1998;26:1136-1137.

57. Gronseth GS, Barohn RJ. Practice parameter: thymectomy for autoimmune myasthenia gravis (an evidence-based review): report of the Quality Standards Subcommittee of the American Academy of Neurology. Neurology. 2000;55:7-15.

58. Soleimani A, Moayyeri A, Akhondzadeh S, Sadatsafavi M, Tavakoli Shalmani H, Soltanzadeh A. Frequency of myasthenic crisis in relation to thymectomy in generalized myasthenia gravis: a 17-year experience. BMC Neurol. 2004;4:12.

59. Thymectomy trial in non-thymomatous myasthenia gravis patients receiving prednisone therapy (ClinicalTrials.gov Identifier NCT00294568).

60. Kas J, Kiss D, Simon V, Svastics E, Major L, Szobor A. Decadelong experience with surgical therapy of myasthenia gravis: early complications of 324 transsternal thymectomies. Ann Thorac Surg. 2001;72:1691-1697.

61. Watanabe A, Watanabe T, Obama T, et al. Prognostic factors for myasthenic crisis after transsternal thymectomy in patients with myasthenia gravis. J Thorac Cardiovasc Surg. 2004; 127:868-876. 